\title{
Dual targeting of monomeric tau and $\alpha$-synuclein aggregation: A new multitarget therapeutic strategy for neurodegeneration
}

\author{
Moustafa T. Gabr ${ }^{*}$ and Francesca Peccatib,c
}

aDepartment of Radiology, Stanford University School of Medicine, Stanford, CA, 94305. Email: gabr@stanford.edu ${ }^{b}$ Center for Cooperative Research in Biosciences (CIC bioGUNE), Basque Research and Technology Alliance (BRTA), Bizkaia Technology Park, Building 801A, 48160 Derio, Spain.

'Departament de Química, Universitat Autònoma de Barcelona, 08193 Bellaterra, Spain

\section{Contents}

Experimental $\quad S 2$

Variation in the emission intensity $\left(\mathrm{I} / \mathrm{I}_{0}\right)$ of ThT in the absence and presence of different $\mathrm{S} 3$

concentrations of MG-2119 in response to aggregation of $\alpha$-syn oligomers

Dose-response curve of MG-2119 binding to $\alpha$-syn oligomers measured by Microscale

Thermophoresis (MSH)

Plot of integrated ITC data for the exothermic interaction between MG-2119 and WT tau

The ten best docked poses of MG-2119 on monomeric tau in blind docking study S5

Cell viability \% of SH-SY5Y cells expressing WT tau as determined by MTT assay in the S6

presence $10 \mu \mathrm{M}$ of MG-2119, squalamine, and MK-886

Cell viability \% of SH-SY5Y cells co-incubated with $\alpha$-syn oligomers $(5 \mu \mathrm{M})$ as determined by

MTT assay in the presence $10 \mu \mathrm{M}$ of MG-2119, squalamine, and MK-886.

PAMPA-BBB permeability $\left(P_{e} \times 10^{-6} \mathrm{~cm} / \mathrm{s}\right)$ of MG-2119 and controls $\quad \mathrm{S} 7$

In vitro pharmacokinetic profile of MG-2119 S7

$\begin{array}{ll}\text { References } & \text { S8 }\end{array}$ 


\section{Preparation of $\alpha$-synuclein oligomers}

$\alpha$-Syn oligomers were prepared as previously described. ${ }^{1}$ In brief, purified $\alpha$-syn was dissolved in PBS to a final concentration of $10 \mathrm{mg} / \mathrm{mL}$ and incubated on ice for 30 minutes while vortexed regularly. After incubation, $\alpha$-syn was centrifuged at $12000 \mathrm{~g}$ for 5 minutes and the supernatant loaded onto a Superdex ${ }^{\mathrm{TM}}$ 200 10/300 GL column (GE Healthcare). The column was eluted with PBS at a flow rate of $0.5 \mathrm{~mL} / \mathrm{minute}$. Oligomers were collected between 18-22 $\mathrm{min}$ and monomers after 38-43 min.

\section{In vitro blood-brain barrier permeability}

The ability of the MG-2119 to penetrate blood-brain barrier was evaluated using a parallel artificial membrane permeation assay (PAMPA) for blood-brain barrier according to the method established by Di et al. $^{2}$ Donepezil and caffeine were used as reference compounds in the PAMA assay. The donor microplate (96-wellfilter plate, PVDF membrane) and the acceptor microplate (indented 96-well plate) were both obtained from Millipore. The acceptor 96-well microplate was filled with $300 \mu \mathrm{L}$ of PBS/EtOH (7:3), and the filter membrane was impregnated with $10 \mathrm{~mL}$ of $\mathrm{PBL}$ in dodecane $(20 \mathrm{mg} / \mathrm{mL})$. The compound was dissolved in DMSO at a concentration of $5 \mathrm{mg} / \mathrm{mL}$ followed diluting 50 -fold with a mixture of PBS/EtOH (7:3) to give a final concentration of $100 \mu \mathrm{g} / \mathrm{mL}$. After that, $200 \mu \mathrm{L}$ of diluted solution and $300 \mu \mathrm{L}$ of PBS/EtOH (7:3) were added to the donor wells. The donor filter plate was placed on the acceptor plate to make the underside of filter membrane in contact with buffer solution. After leaving this sandwich assembly for $16 \mathrm{~h}$ at $25^{\circ} \mathrm{C}$, the donor plate was removed, and the concentrations of tested compound in the acceptor, donor and reference wells were measured with a UV plate reader. Each sample was analyzed three independent runs in four wells.

\section{Pharmacokinetic profiling}

The assays for determining pharmacokinetic profile were performed as previously described. ${ }^{3}$

- Stability in intestinal simulated fluids $\left(t_{1 / 2}, \mathrm{~min}\right)$ was evaluated using erythromycin as a reference compound, $\mathrm{t}_{1 / 2}, \min =475 \mathrm{~min}^{3}$

- Stability in rat liver microsomes was evaluated using verapamil as a reference compound, $\mathrm{t}_{1 / 2}$, $\min =5.9 \mathrm{~min}$.

- Human plasma stability was evaluated using propantheline as a reference compound, $\mathrm{t}_{1 / 2}$, $\min =$ 2.75 min. $^{3}$ 


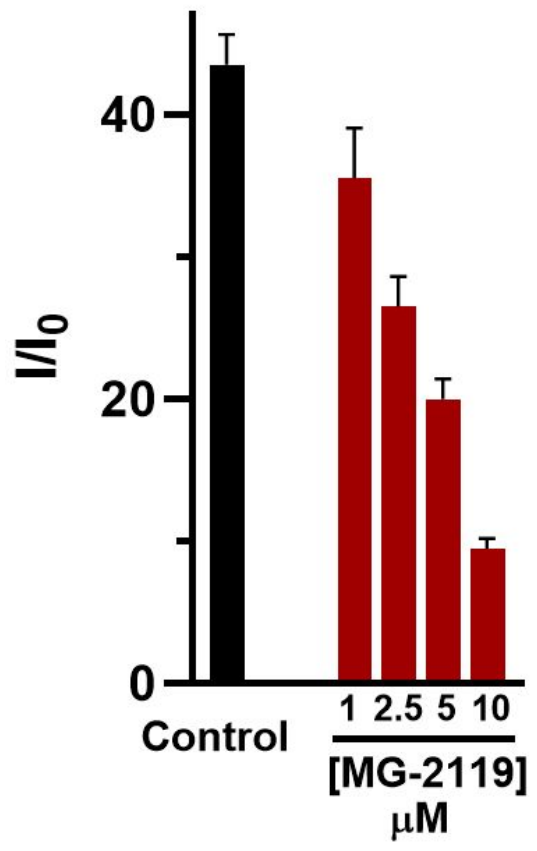

Figure S1. Variation in the emission intensity $\left(\mathrm{I} / \mathrm{I}_{0}\right)$ of ThT in the absence and presence of different concentrations of MG-2119 in response to aggregation of $\alpha$-syn oligomers (100 $\mu \mathrm{M})$ after $12 \mathrm{hr}$ incubation, $\lambda_{\mathrm{ex}}=440 \mathrm{~nm}$, $[\mathrm{ThT}]=5 \mathrm{mM}$.

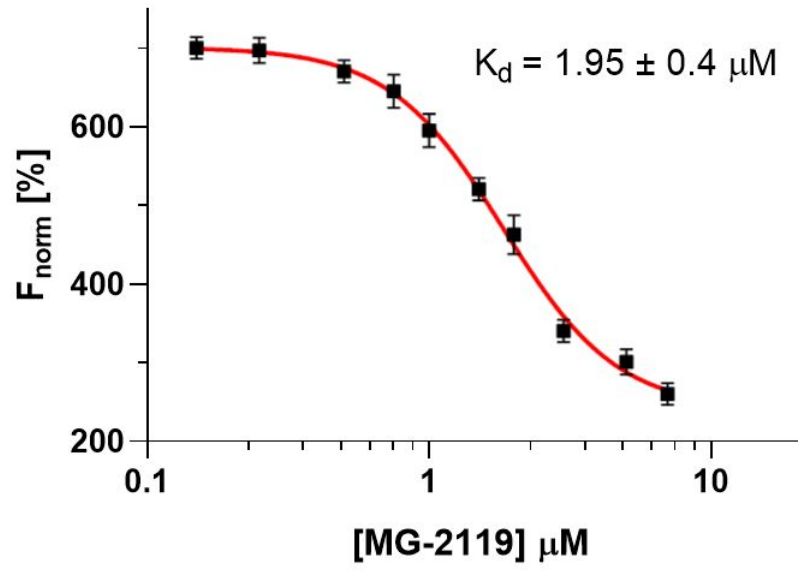

Figure S2. Dose-response curve of MG-2119 binding to $\alpha$-syn oligomers measured by Microscale Thermophoresis (MSH). 


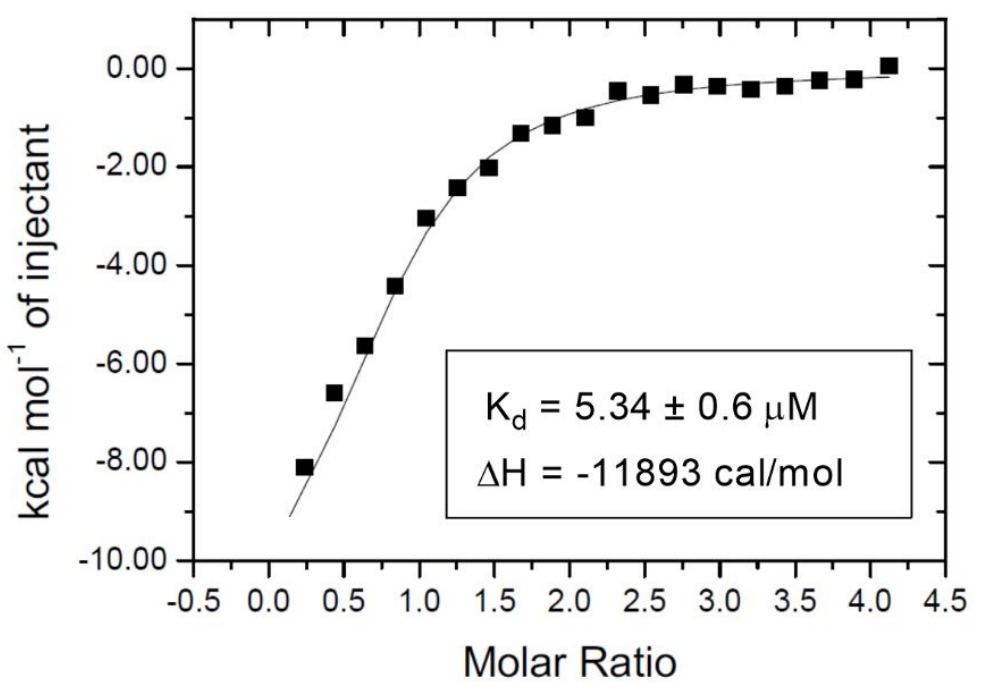

Figure S3. Plot of integrated ITC data for the exothermic interaction between MG-2119 and WT tau (50 $\mu \mathrm{M})$. The solid line represents the best least-squares fit to the data. 


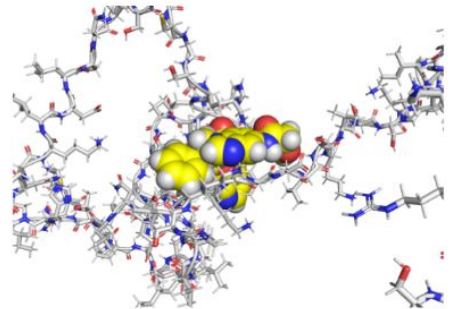

tau27

score: 86.61, resid VAL71, ILE55, TYR68 , LYS52

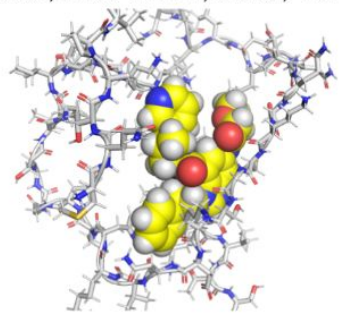

tau44

score: 76.95, resid HIE57, VAL71, LEU7, LYS69

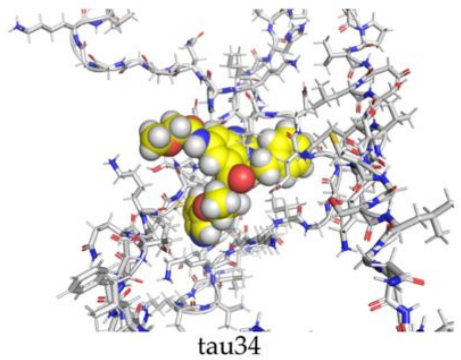

score: 74.93, resid LEU24, ILE18, HIE26, GLU22

4

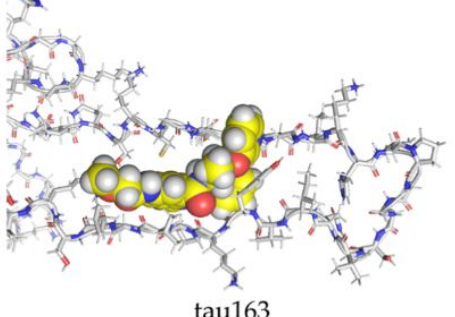

score: 74.18, resid VAL71, TYR68, LYS52, LEU73

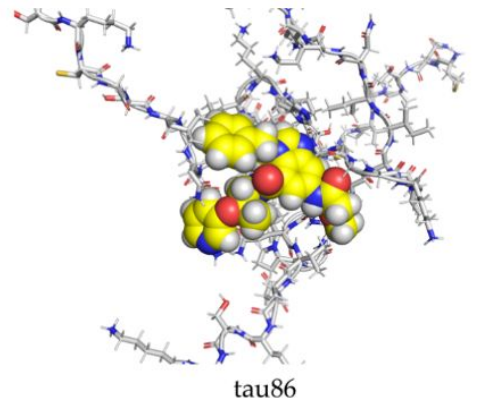

score: 71.35, resid HIE26, GLN34, HIE88, ASN85

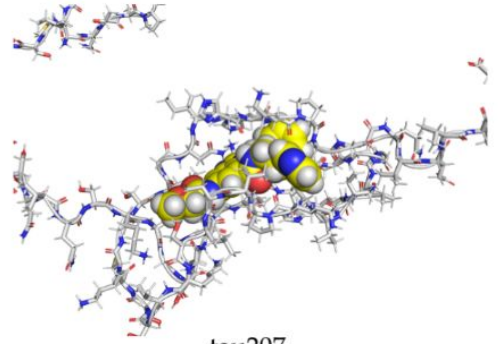

score: 80.23 , resid VAL71, VAL58, ASN 85, LYS89

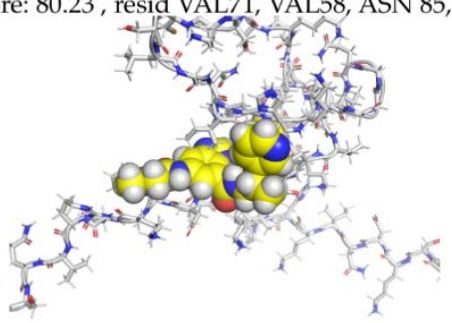

tau144

score: 76.06, resid ILE86, LYS98, PHE104, HIE87

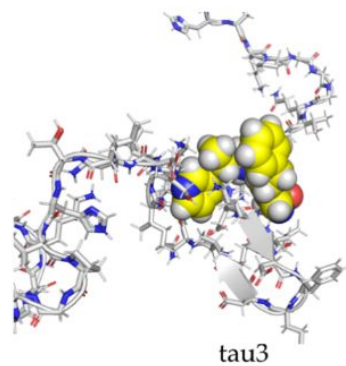

score: 74.27, resid LYS101, LEU102, VAL97, LYS98

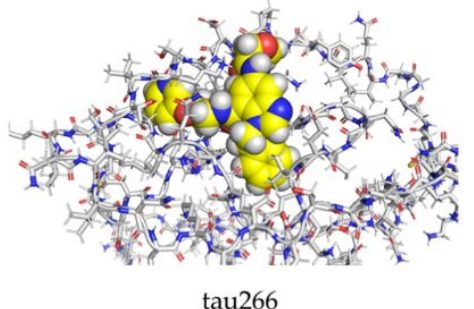

score: 73.95 , residVAL97, LYS89, LEU73, LYS75

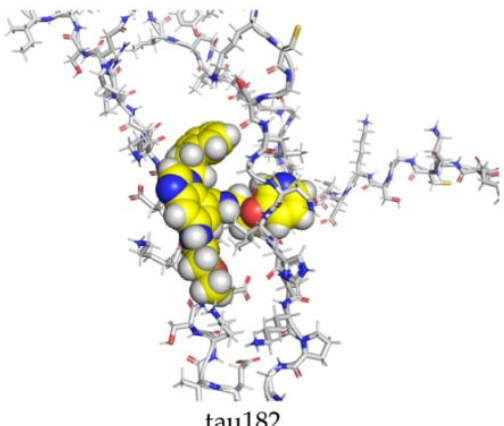

score: 70.83, resid ILE86, LEU83, PHE104, LEU102

Figure S4. The ten best docked poses of MG-2119 on monomeric tau in blind docking study. 


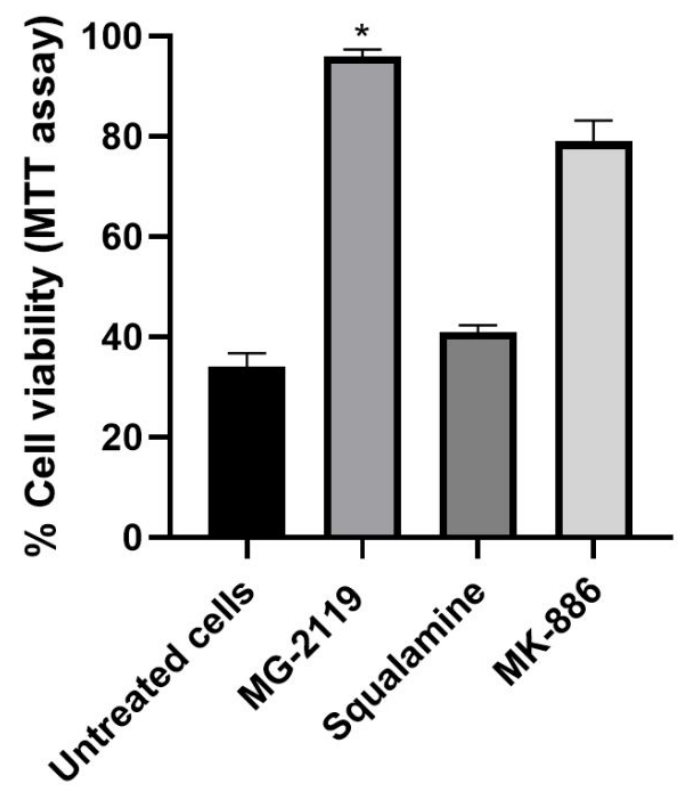

Figure S5. Cell viability \% of SH-SY5Y cells expressing WT tau as determined by MTT assay in the presence $10 \mu \mathrm{M}$ of MG-2119, squalamine, and MK-886. Error bars represent standard deviation $(\mathrm{n}=3, * \mathrm{p}<0.05$ relative to untreated cells).

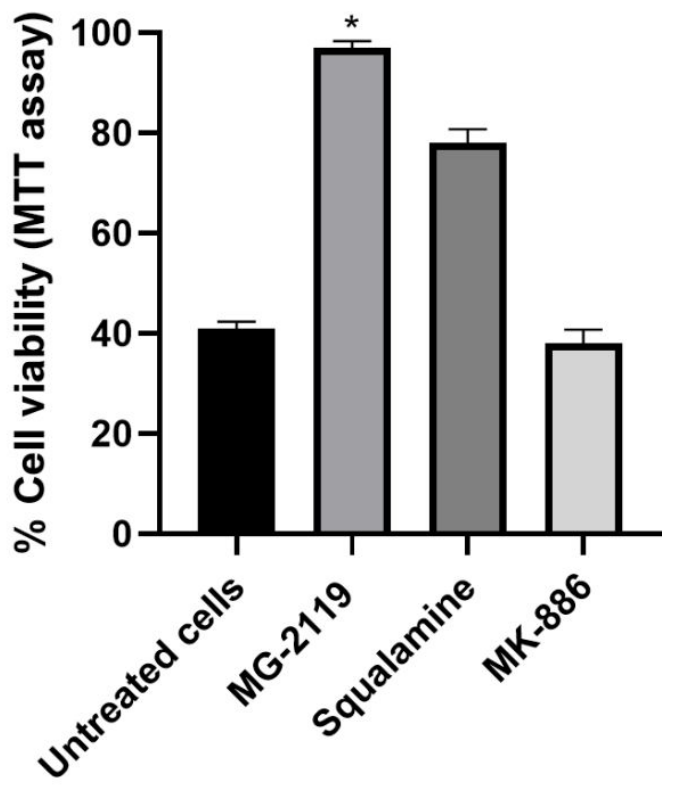

Figure S6. Cell viability \% of SH-SY5Y cells co-incubated with $\alpha$-syn oligomers $(5 \mu \mathrm{M})$ as determined by MTT assay in the presence $10 \mu \mathrm{M}$ of MG-2119, squalamine, and MK-886. Error bars represent standard deviation ( $n=3, * p<0.05$ relative to untreated cells). 
Table S1. PAMPA-BBB permeability $\left(P_{e} \times 10^{-6} \mathrm{~cm} / \mathrm{s}\right)$ of MG-2119 and controls expressed as $P_{e}$ and their predictive penetration to the CNS.

\begin{tabular}{ccc}
\hline Comp. & $\begin{array}{c}\text { Pe } \\
\left(\times 10^{-6} \mathrm{~cm} / \mathrm{s}\right)\end{array}$ & $\begin{array}{c}\text { Predication of } \\
\text { CNS } \\
\text { penetration }\end{array}$ \\
\hline MG-2119 & 29.4 & High \\
\hline Donepezil & 24.2 & High \\
\hline Caffeine & 2.56 & Low \\
\hline
\end{tabular}

Table S2. In vitro pharmacokinetic profile of MG-2119.

\begin{tabular}{lc}
\hline \multicolumn{1}{c}{ Test } & MG-2119 \\
\hline Stability in simulated fluids $\left(\mathrm{t}_{1 / 2}, \mathrm{~min}\right)$ & 285 \\
Gastric $(\mathrm{pH} 1.6)$ & $>500$ \\
Intestinal $(\mathrm{pH} 6.5)$ & \\
Stability in human plasma (\% remaining at 120 & 99.1 \\
min) & \\
CACO cell permeability & 18.9 \\
$\quad$ Papp, ${ }_{A} \rightarrow{ }_{B}\left(10^{-6} \mathrm{~cm} / \mathrm{s}\right)$ & 29.5 \\
$\quad$ Papp, ${ }_{B} \rightarrow{ }_{A}\left(10^{-6} \mathrm{~cm} / \mathrm{s}\right)$ & $>200$ \\
Stability in rat liver microsomes $\left(\mathrm{t}_{1 / 2}, \mathrm{~min}\right)$ & $>50$ \\
Cytotoxicity in $\mathrm{HEL} 299 \mathrm{cells}\left(\mathrm{IC} \mathrm{C}_{50}, \mu \mathrm{M}\right)$ & \\
\hline
\end{tabular}




\section{References}

1. Ruesink, H.; Reimer, L.; Gregersen, E.; Moeller, A.; Betzer, C.; Jensen, P. H., Stabilization of $\alpha$-synuclein oligomers using formaldehyde. PLoS One 2019, 14 (10), e0216764.

2. Di, L.; Kerns, E. H., Profiling drug-like properties in discovery research. Curr. Opin. Chem. Biol. 2003, 7 (3), 402-8.

3. Tosh, D. K.; Finley, A.; Paoletta, S.; Moss, S. M.; Gao, Z.-G.; Gizewski, E. T.; Auchampach, J. A.; Salvemini, D.; Jacobson, K. A., In vivo phenotypic screening for treating chronic neuropathic pain: modification of C2-arylethynyl group of conformationally constrained A3 adenosine receptor agonists. J. Med. Chem. 2014, 57 (23), 9901-9914. 